\section{Measurement of photon sorting at microwave frequencies in a cavity array metasurface}

Eli Lansey, Isroel M. Mandel, Ian R. Hooper, Jonah N. Gollub, Alastair P. Hibbins, David T. Crouse

\begin{abstract}
We present experimental results demonstrating the spatial sorting of incoming radiation in two spectral ranges. A metasurface composed of a periodically patterned metal of subwavelength thickness with dielectric inclusions concentrates and localizes electromagnetic fields near the surface. Light of the separate spectral bands is channeled into different geometrically tuned cavities within each spatially repeating unit cell. Excitation of cavity modes facilitates this simultaneous spatial and spectral selective absorption. The measured reflection and field profiles are presented and show the spectral and spatial selectivity. A method to apply these concepts to split radiation into three spectral bands is also proposed.
\end{abstract}

Index Terms-Metamaterials, Cavity resonators, Periodic structures

\section{INTRODUCTION}

Metasurfaces have the potential to improve the performance of devices in a variety of applications including lensing [1], beam steering [2], solar energy [3], perfect absorbers [4][6], selective emitters [7], [8], and photon sorters [9]-[12]. Metasurfaces are composite films of subwavelength thickness made of a two dimensional array of subwavelength elements or meta-atoms [13]. The materials and geometry of the metaatoms determine how the metasurface interacts with electromagnetic radiation; these meta-atoms can range in size from larger than $\lambda / 4$ to smaller $\lambda / 10$. Cavity array metasurfaces are composed of periodic arrays of dielectric cavities in a metal film of subwavelength thickness. Cavity array metasurfaces have been shown to combine the functionalities of photon sorting, localization, and enhanced dual band absorption [10] and are of interest for use in sensing since the continuous metal metasurface may also serve as an electrical contact unlike multiband absorbers using split ring resonators [4] and local plasmon resonances [6], [11]. In this work we are investigating a novel dual band cavity array metasurface composed of two meta-atom types that are designed to function independently enabling subwavelength localization and near unity absorption of incident electromagnetic wave. Previous work on dual band composites have been done in similar structures such as frequency selective surfaces [14], [15]. The photon sorting capabilities of the dual band cavity array metasurface absorber presented here have been demonstrated in numerical calculations [10]. Here we report on experimental verification of the photon sorting process in a dual band cavity

Eli Lansey and Isroel M. Mandel are with the Department of Physics, The Graduate Center, New York, NY 10016 and City College of the City University of New York, New York, NY 10031

Ian R. Hooper and Alastair P. Hibbins are with the Electromagnetic Materials Group, Department of Physics and Astronomy, University of Exeter, Exeter, Devon EX4, United Kingdom

Jonah N. Gollub is with the Department of Electrical and Computer Engineering, Duke University, Durham, NC 27708

David T. Crouse is with the Department of Electrical Engineering, City College of the City University of New York, New York, NY 10031

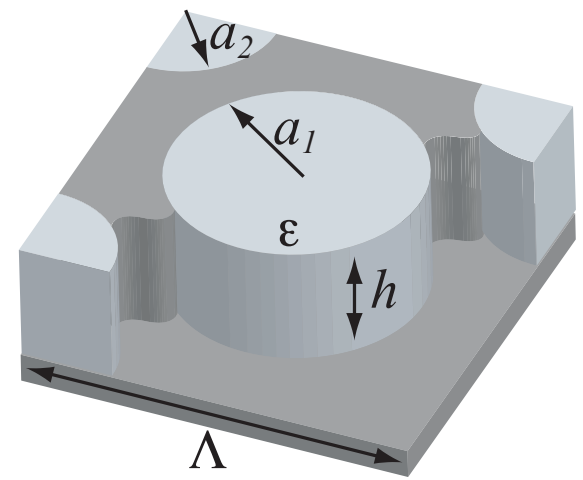

(a) Metasurface unit cell structure

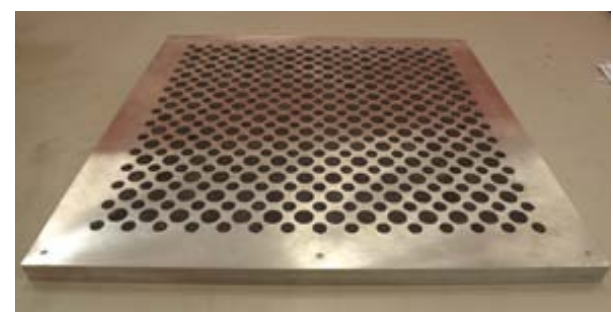

(b) Fabricated metasurface

Fig. 1: The photon sorting metasurface. Each unit cell has two cavities of different radii which resonate at different frequencies.

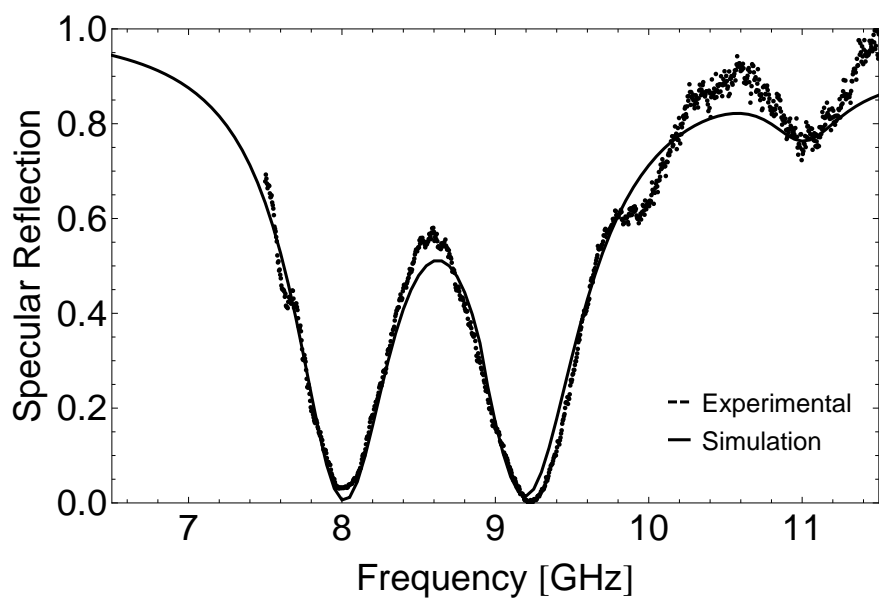

Fig. 2: Experimental and simulated specular reflection intensities from the material surface for s-polarized radiation at a $\theta=$ $17^{\circ}$ angle of incidence. The two dips in the reflected intensity correspond to the two cavity resonances [10].

array metasurface by probing the strength of the electric field above the resonating cavities. We are thus able to directly report the physical mechanism of photon sorting through a direct field measurement instead of only through indirect reflection measurements as is usually done in the literature which masks the underlying process. In addition we present simulated results for a three band cavity array metasurface composed of three meta-atoms per unit cell. 


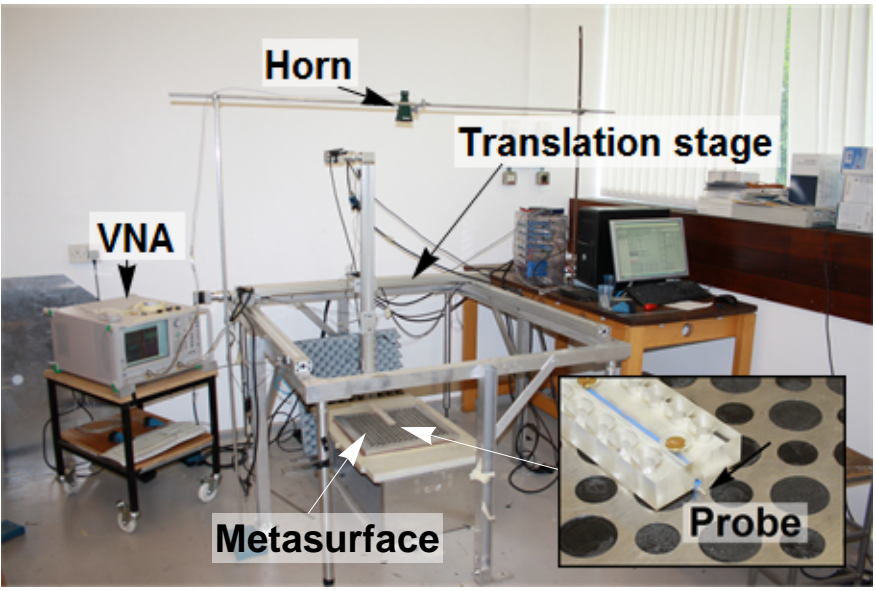

Fig. 3: Experimental setup for measuring the spatial dependence of the electric field strength. The translation stage allows the measuring probe, shown in the inset, to be swept across the top of the metasurface.

\section{Metasurface Design}

In this paper, the structure under investigation is a twodimensional square array of subwavelength cylindrical cavities embedded in a $40 \mathrm{~cm} \times 40 \mathrm{~cm}$ sample of aluminum. Each cavity is filled with an absorbing silicone elastomer dielectric (Sylgard 184), doped with graphite powder (8.36\%), which was selected to maximize the absorption. An Anritsu M4640A vector network analyzer (VNA) was used to record the measured complex S-parameters of the graphite loaded elastomers in an 8-12 GHz waveguide. The dielectric properties of the elastomer composites were subsequently extracted using the Nicolson-Ross-Weir algorithm [16], giving a complex dielectric permittivity of $\epsilon_{r}=4.33+0.22 i$. Each unit cell contains two cavities of different radii and identical heights, arranged in a square lattice, see Fig. 1. The structure's electromagnetic response is due to cavity mode resonances, which are tuned by adjusting the radii and heights of the cavities, as well as the periodicity of the array (see [10] for further discussion). Here the periodicity is $\Lambda=26 \mathrm{~mm}$, cavity radii are $a_{1}=8.03$ $\mathrm{mm}$ and $a_{2}=5.74 \mathrm{~mm}$, and cavity height is $h=7 \mathrm{~mm}$. The simulated and experimentally measured reflection of the metasurface is shown in Fig. 2; the response of the metasurface has peaks in absorption at $8.10 \mathrm{GHz}$ and $9.25 \mathrm{GHz}[10]$ and is polarization independent due to the $90^{\circ}$ rotational symmetry. The experimental setup for obtaining reflection is described below. The electromagnetic response of the metasurface was simulated with periodic boundary conditions on a single unit cell using HFSS a finite element frequency domain solver. In the simulation, the metals were modeled as perfect electric conductors and the measured dielectric permittivity of the graphite doped elastomer was used for the material parameters in the cavities.

\section{RESULTS}

To verify that this device spatially sorts the incident light, we measured the reflection of the sample to determine the resonances and then measured the component of the electric field parallel to the polarization of the incident beam $1 \mathrm{~mm}$ above the structure's surface at the resonant frequencies. To obtain the reflection, two broadband $(8-40 \mathrm{GHz})$ horns from Flann Microwave were placed adjacent to each other approximately $2 \mathrm{~m}$ from the sample such that the angle of incidence was $17^{\circ}$. The horns were attached to an Anritsu M4640A vector network analyser (VNA) which swept a frequency range of $7.5-15 \mathrm{GHz}$ in 2001 points, at a power of $5 \mathrm{~dB}$, and with no averaging. S21 was measured and was normalised to the reflection of a flat aluminium plate in order to determine the absolute reflectivity of the sample, the results of which is compared with the simulated reflection for normal incidence in Fig 2. The agreement with the simulated reflection at normal incidence indicates that the metasurface exhibits angular independence for shallow angles for both polarizations which is a characteristic of such types of cavity arrays. The field profiles were then obtained using the setup shown in Fig. 3. One of the same broadband horns was placed vertically approximately $1.5 \mathrm{~m}$ above the sample. An electrically small probe formed of a $2 \mathrm{~mm}$ long piece of stripped coaxial cable which acts as a small antenna well below any resonant frequencies was attached to a computer controlled translation stage in order to spatially probe the fields $1 \mathrm{~mm}$ above the sample. The measured signal arises from the electric field component along the direction of the exposed probe. The horn and probe were connected to the Anritsu M46040A VNA, with a frequency sweep of 7.5-15 GHz (2001 points) with an IF bandwidth of 1 $\mathrm{kHz}$. The power was $+5 \mathrm{dBm}$, and there was no averaging. S21 was measured as the probe was moved in a plane $1 \mathrm{~mm}$ above the sample, giving a direct measure of the spatial field profile. Figure 4 shows a direct plot of the magnitude of this quantity, where the red color corresponds to the field strength at the 8.1 $\mathrm{GHz}$ resonance and the blue corresponds to the field strength at the $9.25 \mathrm{GHz}$ resonance. The dashed circles mark the approximate edges of the cavities within the metasurface; the enhanced field intensities are localized within the circles. This localization demonstrates the photon sorting property of the metasurface. The vertically-oriented "stripes" of blue between the larger apertures are artifacts of the measurement. Rotating the sample or the source does not change the orientation of the stripes. The likely cause of this effect is the probe wire, which scatters and interferes with the incident radiation. The field maps indicate that the incident light is sorted into different cavities for different spectral bands.

Characterization of the spatially selective absorption of the metasurface cannot be distinguished since the absorption in each individual cavity cannot be directly measured, i.e. the total absorption is inferred from the reflection of the metasurface. However, the absorption in each cavity is dependent on the excitation of a cavity resonance, which results in enhanced field strength in addition to the absorption. Since the tangential component of the electric field is continuous across the interface of the metasurface and the free space above it, a map of the electric field above the metasurface would correspond to a mapping of the fields, and therefore absorption, within the metasurface, i.e. within each cavity. We can therefore use the measured field strength $F$ just above the surface of the metamaterial to calculate an approximate photon 


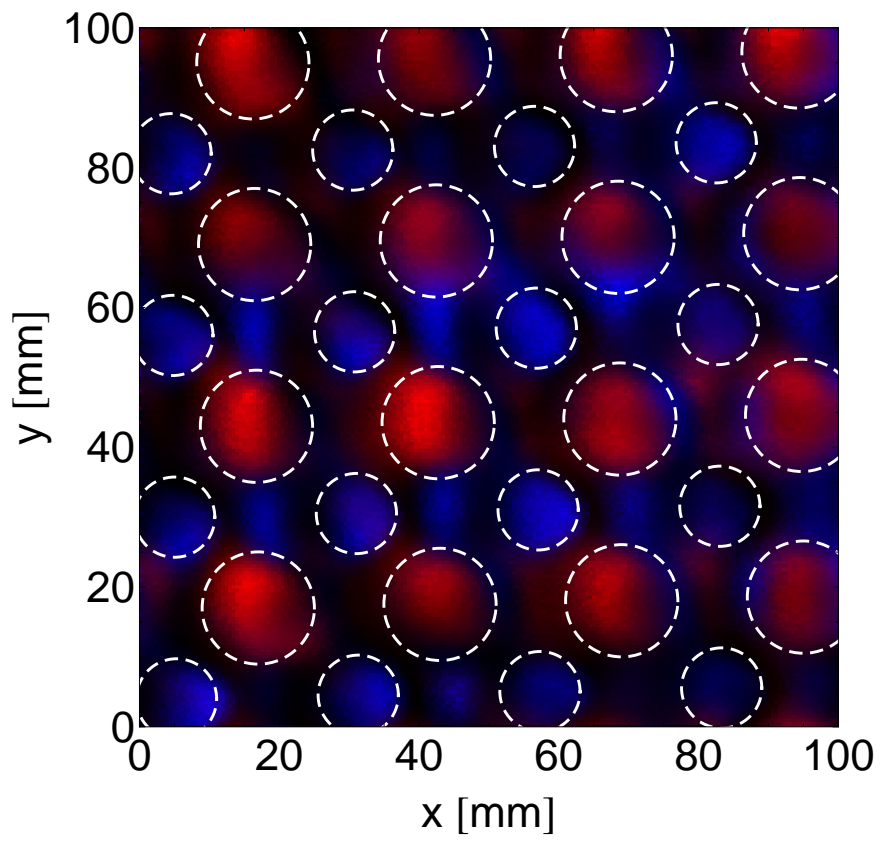

Fig. 4: Pseudo-color plot of the electric field component along the polarized y-direction measured $1 \mathrm{~mm}$ above the metamaterial. The red (blue) color corresponds to the $8.1 \mathrm{GHz}$ $(9.25 \mathrm{GHz})$ resonances, which are overlayed to see the photon sorting. The dashed circles are the approximate edges of the cavities below.

TABLE I: Splitting efficiency from simulated absorption compared to approximate splitting efficiency calculated from measurements.

\begin{tabular}{ccc} 
Frequency $(\mathrm{GHz})$ & Simulated SE & Measured approximate SE \\
\hline 8.1 & 96 & 98 \\
9.25 & 93 & 92
\end{tabular}

sorting efficiency, i.e. the fraction of energy absorbed in the desired cavity [10]. We can estimate the sorting efficiency of the $n t h$ cavity $\left(S E_{n}\right)$ by

$$
S E_{n} \approx \frac{F_{n}}{F_{T}}
$$

where $F_{n}$ is the field strength above cavity $n$ and $F_{T}=F_{1}+$ $F_{2}$ is the total field strength. Figures 5 and 6 shows the average field strength from experiment along lines crossing the large and small cavities. The range of values are shown in the shaded region. Using this approach, and averaging over a few peaks, gives an estimated sorting efficiency of $98 \%$ at $8.1 \mathrm{GHz}$ and $92 \%$ at $9.25 \mathrm{GHz}$. These values compare favorably with the calculated values for the absorption in each cavity obtained from simulation, see Table I. The estimated SE is higher at 9.25 GHz, as this is a measure of field strength, not directly of the absorption; although the fields are higher above the smaller cavity, the absorption may be lower depending on edge effects. Additionally, the value of the SE at this frequency is less precise due to the experimental artifact (i.e./ scattering of the probe wire).

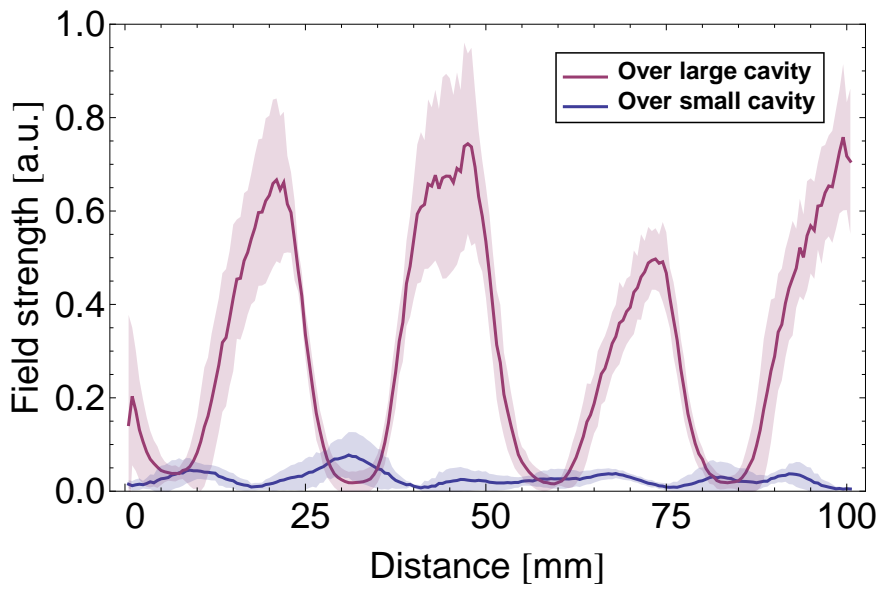

Fig. 5: Experimental measurement of light splitting using field strength above the cavities at $8.1 \mathrm{GHz}$.. The solid lines are the spatial field strength averaged over multiple lines passing through the center of the cavities. Shaded regions show the variation of the average.

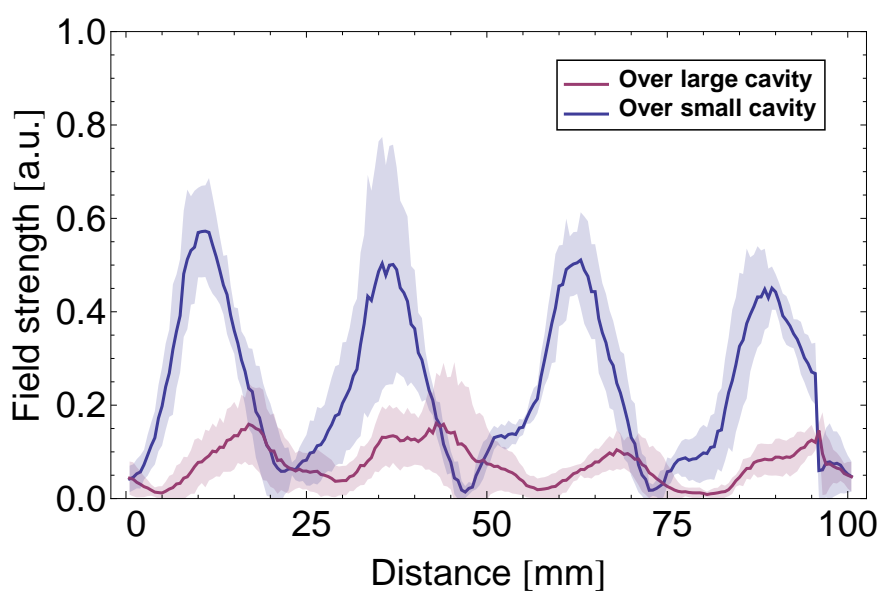

Fig. 6: Experimental measurement of light splitting using field strength above the cavities at $9.25 \mathrm{GHz}$.. The solid lines are the spatial field strength averaged over multiple lines passing through the center of the cavities. Shaded regions show the variation of the average.

TABLE II: Percentage of the total electromagnetic energy absorbed $(E)$ and fractional splitting efficiency $(S E)$ at target frequencies in an optimized three-band structure.

\begin{tabular}{lcccccc} 
& \multicolumn{3}{c}{ Absolute absorption $(\%)$} & \multicolumn{3}{c}{$\mathrm{SE}(\%)$} \\
Frequency & $E_{1}$ & $E_{2}$ & $E_{3}$ & $E_{1} / E_{T}$ & $E_{2} / E_{T}$ & $E_{3} / E_{T}$ \\
\hline $8.05 \mathrm{GHz}$ & 93 & 2 & 1 & 97 & 2 & 1 \\
$9.6 \mathrm{GHz}$ & 6 & 82 & 3 & 6 & 90 & 3 \\
$11.25 \mathrm{GHz}$ & 3 & 3 & 82 & 3 & 4 & 93
\end{tabular}




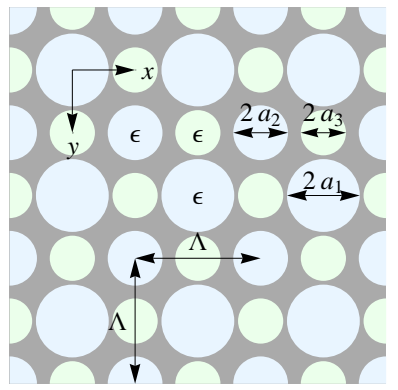

Fig. 7: A top-down view of the three-band light splitting structure. Additional cavities are highlighted in green.

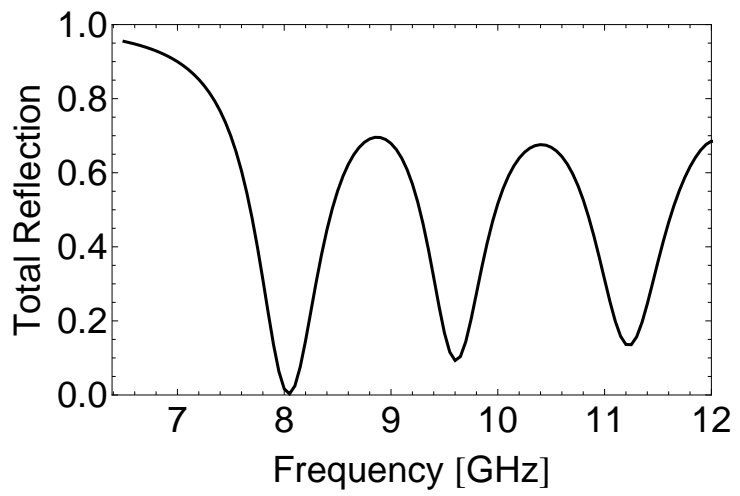

Fig. 8: Simulated reflection from a three-band photon sorting structure.

\section{THREE BAND STRUCTURE}

The patterned material discussed thus far spectrally and spatially sorts microwave radiation into two bands. To numerically investigate a three-band photon sorting structure with three independent resonances, we began with the preceding twoband structure, adjusted various parameters, and added a third cavity, see Fig. 7. More specifically, the simulated device has a periodicity of $\Lambda=24.75 \mathrm{~mm}$, and three cavities with identical heights of $h=8.01 \mathrm{~mm}$, but different radii of $a_{1}=7.25$ $\mathrm{mm}, a_{2}=5.45 \mathrm{~mm}$, and $a_{3}=4.5 \mathrm{~mm}$. In this structure, the dielectric in the cavities is $\epsilon_{r}=4.46+0.05 i$. This device resonates at frequencies of $8.05 \mathrm{GHz}, 9.6 \mathrm{GHz}$, and 11.25 GHz. Fig. 8 shows a plot of the simulated total reflection from this structure as a function of frequency for normally incident radiation of one polarization; there is negligible difference in the reflection response between polarizations, with an absolute difference no greater than $0.6 \%$.

This device absorbs $93 \%$ (82\%) [82\%] of incident radiation at $8.05 \mathrm{GHz}(9.6 \mathrm{GHz})$ [11.25 GHz], with a splitting efficiency of $97 \%(90 \%)$ [93\%]. That is, the fields are channeled to and into the appropriate cavity, where the resonant fields are strongly, and locally absorbed. These results are summarized in Table II. Although this demonstration is for microwave structures, it shows that polarization independent photon sorting is, in principle, possible with three bands, thus increasing the potential efficiency of any device based on this approach. Furthermore, this is a rather simple structure, with identical absorbers in cavities of identical height. The absolute absorption, as well as the SE can be improved by adjusting the dielectrics in each cavity, as well as their heights, independently.

\section{CONCLUSiON}

In conclusion, we have directly demonstrated the photon sorting mechanism for a metasurface that uses cavity metaatom resonances by direct field measurements. By measuring the electric field above the metasurface while under illumination, electric field maps were obtained that displayed the spatial and spectral selectivity of the metasurface absorber showing good agreement with simulated results. We also numerically investigated a three aperture metasurface that is designed to sort and absorb electromagnetic waves in three spectral bands. Multi-band photon sorters of this type may be limited by the amount of cavities that can be placed within a unit cell. In addition, higher order cavity modes may share resonances with other cavities, reducing photon sorting. These issues may be resolved in the future by implementing cavities of different geometries in each unit cell.

\section{ACKNOWLEDGMENT}

This work is supported by the AFOSR Bioenergy project (FA9550-10-1-0350), the NSF Industry/University Cooperative Research Center for Metamaterials (IIP-1068028), and the EPSRC, UK funding through the QUEST project (ref: EP/I034548/1). The design and analysis of the device was done at the The City University of New York, and the fabrication and characterization was done at the Electromagnetics Materials Laboratory at the University of Exeter.

\section{REFERENCES}

[1] F. Aieta, P. Genevet, M. a. Kats, N. Yu, R. Blanchard, Z. Gaburro, and F. Capasso, "Aberration-Free Ultrathin Flat Lenses and Axicons at Telecom Wavelengths Based on Plasmonic Metasurfaces." Nano letters, 12, 4932-4936, (2012).

[2] T. Shegai, S. Chen, V. D. Miljkovic, G. Zengin, P. Johansson, and M. Kall, "A bimetallic nanoantenna for directional colour routing," Nat Commun 2, 481 (2011).

[3] A. V. Kildishev, A. Boltasseva, and V. M. Shalaev, "Planar Photonics with Metasurfaces," Science 339, 1232009,(2013).

[4] H. Tao, C. M. Bingham, D. Pilon, K. Fan, a. C. Strikwerda, D. Shrekenhamer, W. J. Padilla, X. Zhang, and R. D. Averitt, "A dual band terahertz metamaterial absorber," Journal of Physics D: Applied Physics 43, 225102 (2010).

[5] X. Liu, T. Starr, A. F. Starr, and W. J. Padilla, "Infrared Spatial and Frequency Selective Metamaterial with Near-Unity Absorbance,” Physical Review Letters 104, 1-4 (2010).

[6] F. Ding, Y. Cui, X. Ge, Y. Jin, and S. He, "Ultra-broadband microwave metamaterial absorber," Applied Physics Letters 100, 103506 (2012).

[7] X. Liu, T. Tyler, T. Starr, A. F. Starr, N. M. Jokerst, and W. J. Padilla, "Taming the Blackbody with Infrared Metamaterials as Selective Thermal Emitters," Physical Review Letters 107, 045901 (2011).

[8] F. Alves, B. Kearney, D. Grbovic, and G. Karunasiri, "Narrowband terahertz emitters using metamaterial films." Optics express 20, 21025-32 (2012).

[9] E. Laux, C. Genet, T. Skauli, and T. W. Ebbesen, "Plasmonic photon sorters for spectral and polarimetric imaging," Nat Photon 2, 161-164 (2008).

[10] E. Lansey, I. R. Hooper, J. N. Gollub, A. P. Hibbins, and D. T. Crouse, "Light localization, photon sorting, and enhanced absorption in subwavelength cavity arrays." Optics express 20, 24226-36 (2012).

[11] J. Le Perchec, Y. Desieres, N. Rochat, and R. E. de Lamaestre, "Subwavelength optical absorber with an integrated photon sorter," Appl. Phys. Lett. 100, 113304-113305 (2012). 
[12] I. Mandel, J. N. Gollub, I. Bendoym, and D. T. Crouse, "Theory and Design of A Novel Integrated Polarimetric Sensor Utilizing a Light Sorting Metamaterial Grating," IEEE Sensors Journal 13, 618-625 (2013).

[13] C. L. Holloway, E. F. Kuester, J. a. Gordon, J. O'Hara, j. Booth, J. and D. R. Smith,"An Overview of the Theory and Applications of Metasurfaces: The Two-Dimensional Equivalents of Metamaterials,", IEEE Antennas and Propagation Magazine 54, 10-35 (2012).

[14] D. Ramaccia, F. Bilotti, A. Toscano, L. Vegni,"Dielectric-free multiband frequency selective surface for antenna applications,", COMPEL, 32, 1868-1875, (2013).

[15] D. Ramaccia, and A. Toscano,"Inductive tri-band double element FSS for space applications,"PIER C, Vol. 18, 87-101, (2011).

[16] A. M. Nicolson and G. F. Ross,"Measurement of the intrinsic properties of materials by time-domain techniques," IEEE Trans.Instrum. Meas., Vol. 19, (1970). 